\title{
Pelvic inflammatory disease during the post-partum year
}

\author{
BARBARA E. MAHON ${ }^{1,4}$, M'HAMED TEMKIT $^{2}$, JANE WANG ${ }^{3}$, MARC B. ROSENMAN $^{1,3}, \&$ \\ BARRY P. KATZ ${ }^{2}$ \\ ${ }^{1}$ Department of Pediatrics, ${ }^{2}$ Division of Biostatistics, ${ }^{3}$ Regenstrief Institute, Indiana University School of Medicine, IN, USA, \\ and ${ }^{4}$ Departments of Epidemiology and Pediatrics, Boston University Schools of Public Health and Medicine, MA, USA
}

\begin{abstract}
Objective. To investigate the occurrence of, and risk factors for, pelvic inflammatory disease (PID) occurring during the post-partum year.

Methods. Demographic and clinical data for women who delivered a term infant with 5-minute Apgar score $\geqslant 8$ from 1992 through 1999 at a large urban hospital were extracted from an electronic medical record system.

Results. During the study period, 15206 deliveries occurred among 12549 women. PID was diagnosed during the postpartum year of $148(1.0 \%)$ deliveries. In univariate analysis, young age, black race, and both pre-delivery history and postpartum diagnosis of chlamydial and gonococcal infection were associated with PID. In multivariate analysis, only young age and a positive test for gonorrhea before delivery or post-partum were independent predictors of PID.

Conclusions. Pelvic inflammatory disease was diagnosed during the post-partum year in $1 \%$ of women studied. Young maternal age was an important demographic risk factor. Further investigation of post-partum STD acquisition and progression to PID is needed to determine whether women are at increased risk following delivery.
\end{abstract}

Keywords: Pelvic inflammatory disease, post-partum, gonorrhea, chlamydia

\section{Introduction}

Pelvic inflammatory disease (PID), inflammation of the upper female genital tract often associated with chlamydial or gonococcal infection, is a major clinical and public health problem. About 1.2 million episodes of acute PID leading to 130000 hospitalizations are currently estimated to occur annually in the United States [1]. The impact of PID exceeds the burden of acute illness, because PID also causes serious long-term consequences, including ectopic pregnancy, infertility, and chronic abdominal or pelvic pain [2]. Direct medical costs for PID and its sequelae are estimated to exceed $\$ 1.8$ billion annually [1].

The rationale for screening women for chlamydial and gonococcal infection rests in large part on the potential of such screening programs to reduce the incidence of PID [3]. Since the incidence of chlamydia- or gonorrhea-associated PID depends both on the probability of acquisition of infection with these organisms and on the probability of progression of lower genital tract infection to PID, factors that increase the probability of either chlamydial or gonococcal infection will be expected to lead to greater incidence. Existing screening recommendations focus on screening women at high risk for STD acquisition-sexually active young women, older women with personal risk factors such as new or multiple sexual partners, and women from populations with high rates of chlamydial and gonococcal infection [4]. Economic studies have suggested that screening for chlamydia is costeffective at prevalence of at least 3.1\% [5]. However, if certain populations of women were at higher risk for development of PID, given lower genital tract infection, then screening such populations would be cost-effective at lower prevalence.

Two recent studies have documented substantial rates of chlamydial and gonococcal infection in postpartum women, most of whom had negative tests for these organisms before delivery, raising the question of whether late pregnancy and the post-partum period may be a period of increased risk for acquisition of these STDs [6,7]. Whether chlamydial or gonococcal infections acquired during the postpartum period are more likely to progress to PID than infections acquired at other times is not known.

Correspondence: Barbara E. Mahon, MD, MPH, Department of Epidemiology, Boston University School of Public Health, 715 Albany Street, T3E, Boston, MA 02118, USA. Tel: (617) 638-6736. Fax: (617) 638-4458. E-mail: mahonbe@bu.edu 
However, post-partum endometritis due to preexisting chlamydial and gonococcal infection is a well recognized entity [8], and it is plausible that the cervical, uterine, and immune changes of pregnancy and delivery, which resolve over six weeks to three months after delivery [9], could facilitate progression. In one rigorous study controlling for age, parous women were at considerably higher risk of PID than nulliparous women [10], but this study did not examine time from delivery to PID; to our knowledge, no epidemiologic studies of PID have examined the role of recent childbirth in PID. To explore the epidemiology of PID occurring during the post-partum year, we used an electronic medical record system to conduct a cohort study of a large population of women who delivered an infant in an urban Midwestern hospital from 1992 through 1999.

\section{Materials and methods}

The study was approved by the Institutional Review Board of Indiana University, Purdue University, Indianapolis. The study population included all women who delivered an infant of 37 to 44 weeks gestational age with a 5-minute Apgar score of $\geqslant 8$ from January 1, 1992 through December 31, 1999 at Wishard Memorial Hospital, a large urban hospital that serves a diverse but predominantly inner-city population in Indianapolis.

Clinical data were extracted from the Regenstrief Medical Records System (RMRS), a comprehensive electronic medical records system that gathers and stores data from Wishard Memorial Hospital and its affiliated outpatient sites [11]. Age at delivery, gravidity, parity, race as recorded by a nurse at the first prenatal visit, mode of delivery, and the results of all tests for gonorrhea and chlamydia performed before, during, and after pregnancy were extracted. Throughout the study period, prenatal chlamydia and gonorrhea testing was performed routinely at entry to prenatal care and was repeated as clinically indicated. Post-partum chlamydia and gonorrhea testing was performed routinely at the post-partum medical evaluation (about $55 \%$ of women were tested within 12 weeks of delivery in another study in this site) and in other settings as clinically indicated.

To identify episodes of PID, RMRS inpatient and outpatient files were searched for occurrences of pertinent International Classification of Diseases, Ninth Revision (ICD-9) codes [12] from January 1, 1992 through December 31, 2000, a year after the last study delivery. To exclude infectious complications of delivery, only episodes occurring $>30$ days after delivery were included. A diagnosis of PID was defined as the occurrence of an ICD-9 code for acute adnexitis and oophoritis (614.0); salpingitis and oophoritis of unspecified duration (614.2); acute parametritis and pelvic cellulitis (614.3); pelvic peritonitis (614.5); unspecified inflammatory disease of female pelvic organs and tissues, which includes the diagnosis 'pelvic inflammatory disease' (614.9); gonococcal perihepatitis/Fitz-Hugh-Curtis syndrome (098.86); chlamydial perihepatitis (099.56); or episodes coded as either abdominal pain (625.9) or pelvic pain (789.0) occurring within two days of a positive test for gonorrhea or chlamydia.

For each delivery, ICD-9 codes that occurred from 31 to 365 days after delivery were included. However, if the woman became pregnant again during the post-partum year, her observations were censored at the estimated date of conception, which was determined by subtracting the gestational age at subsequent delivery from the date of that delivery. Also, to attempt to exclude person-time for women who did not obtain follow-up care at RMRS sites, observations for women who had no visits recorded in the RMRS after the post-partum year were censored on the date of their last RMRS entry during the post-partum year.

Written medical records of all women with more than one diagnosis of PID were reviewed to determine whether the multiple codes represented multiple episodes of illness; dates that represented follow-up examinations for earlier episodes were not included. These records as well as records of all women hospitalized within seven days before or after the date of the ICD-9 code, of all women who were included as PID cases on the basis of ICD-9 codes 625.9 or 789.0 , and of additional women chosen at random were also reviewed to validate the diagnostic coding.

Statistical analyses were conducted with SAS version 8.2 (SAS Institute, Cary, NC) and S-Plus version 6.1 (Seattle, WA). Race was dichotomized as black versus non-black, based on previous work in this Indianapolis population that showed higher prevalence of chlamydial and gonococcal infection among women identified as black and uniformly lower prevalence within non-black groups. Parity was dichotomized as 0 versus $\geqslant 1$, and gravidity as 1 versus $\geqslant 2$. Post-partum and pre-delivery (including both pre-pregnancy and prenatal) chlamydial and gonococcal infection status were classified as 'ever positive', 'never positive', or 'never tested' for each time period. For women diagnosed with post-partum PID, only tests for chlamydia and gonorrhea that were performed on or before the date of PID diagnosis were included. $p$-Values for measures of association in a bivariate analysis of each predictor variable and post-partum PID were obtained using Generalized Estimating Equations to adjust for correlated observations due to multiple deliveries in some women [13]; predictor variables with 
$p$-values $<0.3$ were retained for further investigation in a multivariate survival model.

Survival analysis of time to PID after delivery was conducted using an Andersen-Gill model, which adjusts for multiple events occurring in the same subject [14]. This model was developed to examine the effects of chlamydial and gonococcal infection on the time to occurrence of PID, after adjustment for relevant covariates. Infection status for both organisms, pre-delivery and post-partum, were fixed in the model. Age, gravidity, parity, mode of delivery, and race were also included in an initial model. Records with missing data on variables included in the model and records for women whose observation time was censored at $\leqslant 30$ days postpartum were excluded. Stepwise backward selection of variables was used to obtain the final model.

\section{Results}

During the study period, 15206 eligible deliveries occurred among 12549 women. Table I summarizes data on these deliveries. Chlamydial and gonococcal infection were common in this population, with at least one positive pre-delivery (before or during pregnancy) test for chlamydia for 4028 (26.5\%) deliveries and for gonorrhea for 2244 (14.8\%) deliveries and with lower but still substantial numbers of positive tests during the post-partum year. Women who were 'never positive' for these STDs were similar demographically and in rates of post-partum PID to women who were 'never tested', so these categories were combined for further analysis.

A full year of observation was available for 10906 $(71.7 \%)$ deliveries; observation was censored between 30 and 364 days for 3316 (21.8\%). Observation was censored $\leqslant 30$ days after delivery for $984(6.5 \%)$ deliveries; since our definition of PID excluded episodes occurring this soon after delivery, these deliveries could not have contributed PID episodes and were excluded from PID-related analyses. Deliveries with post-partum observation censored $\leqslant 30$ days after delivery were somewhat more likely to have been to non-black women, to multiparous women, and to women without a previous positive gonorrhea or chlamydia test.

All of the PID-defining ICD-9 codes were found in the RMRS. Within the post-partum year for study women, ICD-9 code 614.9 (includes 'pelvic inflammatory disease') occurred 129 times, and 614.0 (acute adnexitis and oophoritis) and 614.2 (salpingitis and oophoritis of unspecified duration) occurred three times each. Code 625.9 (abdominal pain) occurred within two days of a positive test for gonorrhea or chlamydia 11 times, and 789.0 (pelvic pain) five times; of these episodes, half were
Table I. Characteristics of deliveries of infants at gestational age 37 to 44 weeks with a 5-minute Apgar score of $\geqslant 8$ from January 1 , 1992 through December 31, 1999 at Wishard Memorial Hospital, Indianapolis, IN.

\begin{tabular}{|c|c|c|}
\hline \multicolumn{3}{|l|}{ Characteristics of deliveries } \\
\hline Total number of deliveries & 15206 & \\
\hline \multicolumn{3}{|l|}{$\begin{array}{l}\text { Number of mother's deliveries } \\
\text { during study period, } n(\%)\end{array}$} \\
\hline 1 & 10276 & $(67.6)$ \\
\hline 2 & 3846 & (25.3) \\
\hline$\geqslant 3$ & 1084 & $(7.1)$ \\
\hline $\begin{array}{l}\text { Mother's age in years at delivery, } \\
\text { median (range) }\end{array}$ & 22 & $(12-47)$ \\
\hline \multicolumn{3}{|l|}{ Race, $n(\%)$} \\
\hline Black & 8439 & $(55.5)$ \\
\hline Non-black & 6726 & $(44.2)$ \\
\hline Not recorded & 41 & $(0.3)$ \\
\hline \multicolumn{3}{|l|}{$\begin{array}{l}\text { Gravidity, recorded at onset } \\
\text { of prenatal care, } n(\%)\end{array}$} \\
\hline 1 & 4030 & (26.5) \\
\hline$\geqslant 2$ & 9111 & $(59.9)$ \\
\hline Not recorded & 2065 & (13.6) \\
\hline \multicolumn{3}{|l|}{$\begin{array}{l}\text { Parity, recorded at onset } \\
\text { of prenatal care, } n(\%)\end{array}$} \\
\hline 0 & 4773 & (31.4) \\
\hline$\geqslant 1$ & 7864 & $(51.7)$ \\
\hline Not recorded & 2569 & $(16.9)$ \\
\hline \multicolumn{3}{|l|}{ Mode of delivery, $n(\%)$} \\
\hline Caesarean & 2176 & (14.3) \\
\hline Vaginal & 12995 & $(85.5)$ \\
\hline Not recorded & 35 & $(0.2)$ \\
\hline \multicolumn{3}{|l|}{$\begin{array}{l}\text { Positive STD test before or during } \\
\text { pregnancy, } n(\%)\end{array}$} \\
\hline Chlamydia & 4028 & $(26.5)$ \\
\hline Gonorrhea & 2244 & $(14.8)$ \\
\hline \multicolumn{3}{|l|}{$\begin{array}{l}\text { Positive STD test during years } \\
\text { post-partum } *, n(\%)\end{array}$} \\
\hline Chlamydia & 700 & $(4.6)$ \\
\hline Gonorrhea & 337 & $(2.2)$ \\
\hline
\end{tabular}

*For women with PID, only tests that preceded or coincided with the PID episode were included.

associated with gonorrhea and half with chlamydia. Thus, a total of 151 episodes met the definition of a diagnosis of PID. One woman had PID following two different deliveries, and three women had two separate episodes of PID in the year following a delivery. Thus, PID was diagnosed during the postpartum year of $148(1.0 \%)$ deliveries. Figure 1 shows the incidence rate by post-partum month of these illnesses.

In bivariate analysis, young age was strongly associated with post-partum PID; the odds of post-partum PID decreased by $6 \%$ for each additional year of age $(p<0.0001)$. Table II shows the association of other demographic and clinical variables with post-partum PID; black race and both pre-delivery history and post-partum diagnosis of chlamydial and gonococcal infection were statistically significantly associated with PID. In multivariate 


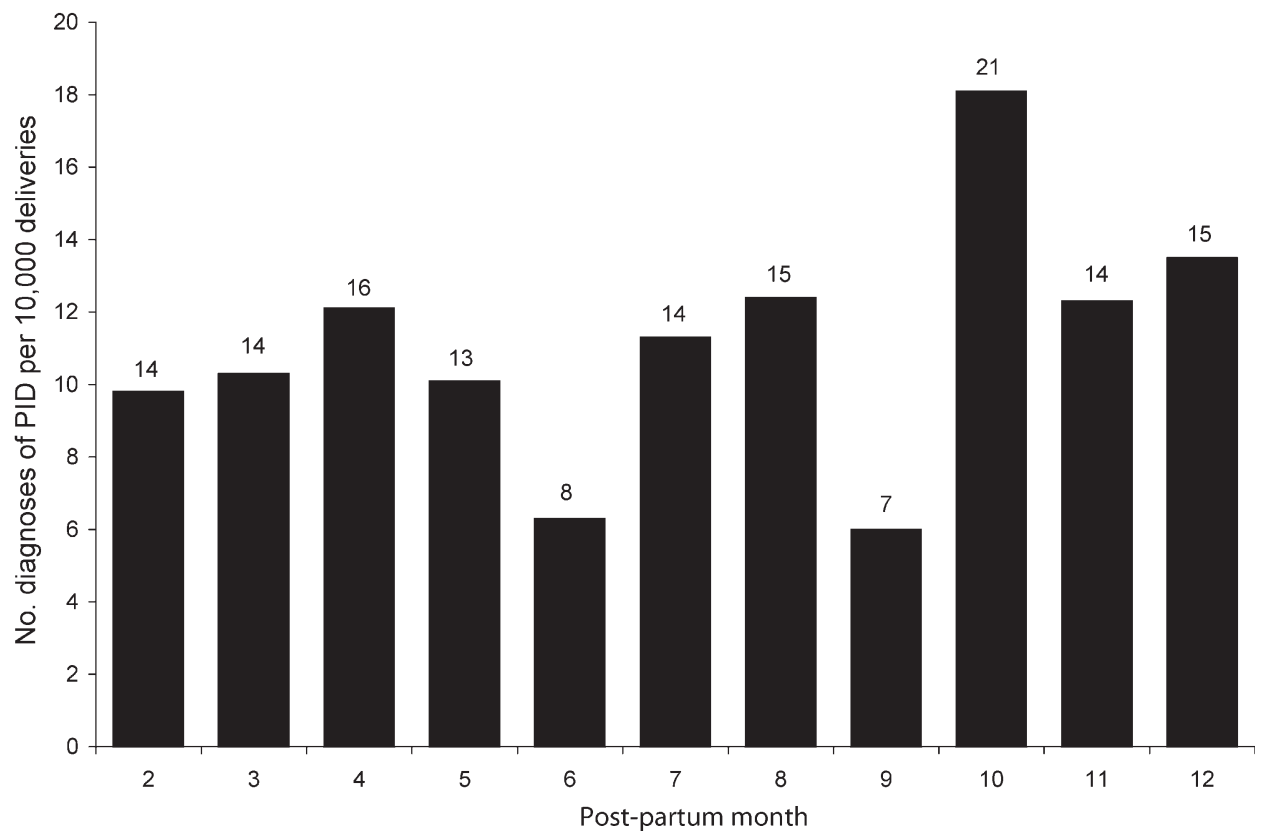

Figure 1. Incidence rate (bar) and number (above bar) by post-partum month of episodes of PID diagnosed during the post-partum year (excluding the first post-partum month).

survival analysis, young age and a positive test for gonorrhea either before delivery or post-partum were independent predictors of time to PID, but black race and positive tests for chlamydia were not. The final model is shown in Table III.

In all, 31 women were hospitalized during the post-partum illness episode. Review of these medical records showed that $22(71 \%)$ women had a discharge diagnosis of PID; two who were admitted with diagnoses of PID had a discharge diagnosis of pyelonephritis; one each had appendicitis, cholelithiasis, gastroenteritis, and ureteral calculus; and records for three women were not available. An additional 53 women treated as outpatients were selected for record review; records of 43 (81\%) were available. Many records were missing key clinical information, such as white blood cell count, used in the CDC definition of PID, but uterine, adnexal, or cervical motion tenderness was documented in $36(84 \%)$ of these records, and, among the other 7 records, 3 documented both abdominal pain and concurrent infection with chlamydia alone or together with gonorrhea.

\section{Discussion}

This study shows that, in this urban Midwestern site with a high rate of prenatal STDs, PID was diagnosed during the year following $1 \%$ of deliveries. To our knowledge, this is the first epidemiologic study of PID to focus on post-partum women beyond the immediate post-partum days and weeks. Although an annual rate of PID of $1 \%$ may seem high, it is difficult for two reasons to compare this rate to that in other populations. First, methodological issues and uncertainty in the clinical diagnosis of PID have produced a wide range of estimated PID frequency in the United States [2]. Second, our study examined only post-partum women, therefore we do not know how the PID rate we observed would have compared to the rate among an otherwise-similar group of women who had not delivered in the previous year. We believe that the value of this study lies in calling attention to the question of whether STD acquisition and progression during pregnancy and the post-partum period may increase the risk of PID after childbirth. This question should be addressed in prospective studies.

Consistent with studies of other chlamydia- and gonorrhea-related outcomes, our multivariate model of time to PID showed that young age was a strong independent risk factor for PID during the year after delivery [15-17]. A positive test for gonorrhea, either post-partum or before or during pregnancy was also independently associated with PID during the postpartum year. Although we do not have data to document that pre-delivery infections were treated, clinical practice is to treat these infections at the time of diagnosis and to confirm that treatment has been completed at subsequent prenatal visits and at delivery. Therefore, our finding of an association of post-partum PID with pre-delivery gonococcal infection is consistent with other studies that have shown that women with STDs often become reinfected after treatment [18-23]. The finding that positive chlamydia tests were not independently associated with PID may reflect the fact that 
chlamydia tends to cause a more indolent infection than gonorrhea, and therefore may be less likely to result in an acute illness recognized as PID.

This study is limited by its reliance on existing medical records and ICD-9 codes to define episodes of PID. Because of this method of data collection, we were unable to use strict clinical and laboratory criteria to define PID, as has been proposed to

Table II. Association of demographic and clinical characteristics with PID diagnosed during the post-partum year. Observations with missing values are excluded.

\begin{tabular}{|c|c|c|c|}
\hline Characteristics & $\begin{array}{l}\text { PID diagnoses } \\
\text { per } 1000 \\
\text { deliveries }\end{array}$ & $\mathrm{RR}$ & $p$-value* \\
\hline \multicolumn{4}{|l|}{ Race } \\
\hline Black & 13.0 & 2.19 & \multirow[t]{2}{*}{$<0.0001$} \\
\hline Non-black & 5.9 & Ref & \\
\hline \multicolumn{4}{|l|}{ Gravidity } \\
\hline 1 & 9.7 & 1.05 & \multirow[t]{2}{*}{0.97} \\
\hline$\geqslant 2$ & 10.2 & Ref & \\
\hline \multicolumn{4}{|l|}{ Parity } \\
\hline 0 & 10.1 & 0.97 & \multirow[t]{2}{*}{0.82} \\
\hline$\geqslant 1$ & 9.8 & Ref & \\
\hline \multicolumn{4}{|c|}{ Mode of delivery } \\
\hline Caesarean & 9.6 & 0.96 & \multirow[t]{2}{*}{0.79} \\
\hline Vaginal & 10.0 & Ref & \\
\hline \multicolumn{4}{|l|}{$\begin{array}{l}\text { Pre-delivery } \\
\text { chlamydia }\end{array}$} \\
\hline Yes & 17.4 & 2.40 & \multirow[t]{2}{*}{$<0.0001$} \\
\hline No & 7.2 & Ref & \\
\hline \multicolumn{4}{|l|}{$\begin{array}{l}\text { Pre-delivery } \\
\text { gonorrhea }\end{array}$} \\
\hline Yes & 26.3 & 3.70 & \multirow[t]{2}{*}{$<0.0001$} \\
\hline No & 7.1 & Ref & \\
\hline \multicolumn{4}{|l|}{$\begin{array}{l}\text { Post-partum } \\
\text { chlamydia }\end{array}$} \\
\hline Yes & 25.7 & 2.81 & \multirow[t]{2}{*}{0.002} \\
\hline No & 9.2 & Ref & \\
\hline \multicolumn{4}{|l|}{$\begin{array}{l}\text { Post-partum } \\
\text { gonorrhea }\end{array}$} \\
\hline Yes & 97.9 & 12.34 & \multirow[t]{2}{*}{$<0.0001$} \\
\hline No & 7.9 & Ref & \\
\hline
\end{tabular}

${ }^{*} p$-Values were obtained using generalized estimating equations to adjust for correlated observations due to multiple deliveries in some women. RR, risk ratio; Ref, reference. improve the specificity of the diagnosis of PID [4,24]. However, review of the complete written medical records of women who were hospitalized for their illness showed that $71 \%$ had a discharge diagnosis of PID and that, among women treated as outpatients, the vast majority had symptoms consistent with PID, including cervical, uterine, or adnexal tenderness in $84 \%$, consistent with CDC's recommendation for minimum criteria for treatment for PID [4]. Even if some of the illnesses coded as PID would not have met a strict clinical case definition, it seems likely that many of them would have.

Several aspects of the study design may have tended to decrease the observed incidence of PID. First, deliveries in which the infant was born prematurely or with a 5-minute Apgar score $<8$ were excluded, because of the complex interaction of existing maternal infections with these outcomes. Although few deliveries were excluded for these reasons, it is plausible that these mothers could have been at higher risk for subsequent PID. Similarly, one could argue that censoring women who did not return for follow-up at RMRS sites, although prudent to ensure that women were under observation, may have excluded women who were less reliable users of healthcare and perhaps were at higher risk for PID.

Few studies have examined the role of childbearing - pregnancy, delivery, and the postpartum period-in STD acquisition or in the development of PID. However, many aspects of this period-decreased coital activity or abstinence during late pregnancy, the possibility of increased extra-dyadic sexual relationships by male partners, frequent early resumption of coitus during the post-partum period, and possible increased biological susceptibility due to the physical changes to the genital tract associated with delivery-suggest that it could represent a time of increased risk. Our previous study in this setting showed an unexpectedly high rate of new chlamydial and gonococcal infections diagnosed during the 12 weeks post-partum [7], as did a study of post-partum adolescents in Connecticut [6]. The high absolute

Table III. Survival analysis of time to PID after delivery, examining the effects of chlamydial and gonococcal infection on the time to occurrence of PID after adjustment for covariates $\left(n=14156^{*}\right)$.

\begin{tabular}{|c|c|c|c|}
\hline Variable & Hazard Ratio & $95 \%$ Confidence Interval & $p$-Value \\
\hline $\mathrm{Age}^{\dagger}$ & 0.954 & $0.917,0.991$ & 0.02 \\
\hline Predelivery C. trachomatis $^{\ddagger}$ & 1.375 & $0.919,2.058$ & 0.12 \\
\hline Predelivery $N$. gonorrhoea $e^{\ddagger}$ & 2.028 & $1.321,3.113$ & 0.001 \\
\hline Post-partum C. trachomatis $^{\S}$ & 1.132 & $0.574,2.232$ & 0.72 \\
\hline Post-partum $N$. gonorrhoeae ${ }^{\S}$ & 8.525 & $4.902,14.828$ & $<0.0001$ \\
\hline
\end{tabular}

C. trachomatis, Chlamydia trachomatis; N. gonorrhoeae, Neisseria gonorrhoeae. *Records with missing data on variables included in the model and records censored at $\leqslant 30$ days post-partum were excluded; ${ }^{\dagger}$ Hazard ratio for each additional year of age; ${ }^{\ddagger}$ Defined as ever positive versus never positive/not tested for the pre-pregnancy and prenatal period; ${ }^{\S}$ Defined as ever positive versus never positive/not tested during the post-partum period. For women with PID, only tests that preceded or coincided with the PID episode were included. 
rate of PID that we found is consistent with, but without a non-post-partum comparison group does not specifically support, the hypothesis that risk for PID is increased after delivery. Future research to explore the role of pregnancy, childbirth, and the post-partum period in STD acquisition and in progression to PID is needed. If increased risk is confirmed, then it will be important to investigate biological and behavioral reasons and to explore prevention strategies.

\section{Acknowledgments}

This study was supported by the Indiana University Center for Excellence in Women's Health.

\section{References}

1. Rein DB, Kassler WJ, Irwin KL, Rabiee L. Direct medical cost of pelvic inflammatory disease and its sequelae: decreasing, but still substantial. Obstet Gynecol 2000;95:397-401.

2. Westrom L, Eschenbach D. Pelvic inflammatory disease. In: Holmes KK, Sparling PF, Mardh P-A, et al., editors. Sexually transmitted diseases. New York: McGraw-Hill; 1999. pp 783-809.

3. Scholes D, Stergachis A, Heidrich FE, Andrilla H, Holmes KK, Stamm WE. Prevention of pelvic inflammatory disease by screening for cervical chlamydial infection. $\mathrm{N}$ Engl J Med 1996;334:1362-1366.

4. Centers for Disease Control and Prevention. Sexually transmitted diseases treatment guidelines 2002. MMWR Morb Mortal Wkly Rep 2002;51(RR-6).

5. Honey E, Augood C, Templeton A, Russell I, Paavonen J, Mardh PA, Stary A, Stray-Pederson B. Cost effectiveness of screening for Chlamydia trachomatis: a review of published studies. Sex Transm Infect 2002;78:406-412.

6. Ickovics J, Niccolai L, Lewis J, Kershaw T, Ethier K. High postpartum rates of sexually transmitted infections among teens: pregnancy as a window of opportunity for prevention. Sex Transm Infect 2003;79:469-473.

7. Mahon B, Rosenman M, Graham M, Fortenberry J. Postpartum Chlamydia trachomatis and Neisseria gonorrhoeae infections. Am J Obstet Gynecol 2002;186:1320-1325.

8. Watts DH, Brunham RC. Sexually transmitted diseases, including HIV infection in pregnancy. In: Holmes KK, Sparling PF, Mardh P-A, et al., editors. Sexually transmitted diseases. New York: McGraw-Hill; 1999. pp 1089-1132.

9. Ferris AM, Reece EA. Puerperium and lactation. In: Reece EA, Hobbins JC, editors. Medicine of the fetus and mother. Philadelphia: Lippincott-Raven; 1999. pp 1653-1670.
10. Jossens MOR, Eskenazi B, Schachter J, Sweet RL. Risk factors for pelvic inflammatory disease: a case control study. Sex Transm Dis 1996;23:239-247.

11. McDonald CJ, Tierney WM, Overhage JM, Martin DK, Wilson GA. The Regenstrief Medical Record System: 20 years of experience in hospitals, clinics, and neighborhood health centers. MD Comput 1992;9:206-217.

12. American Medical Association. International classification of diseases, ninth revision, clinical modification (ICD-9-CM). Salt Lake City, UT: Medicode Publications; 1996.

13. Zeger S, Liang K. Longitudinal data analysis for discrete and continuous outcomes. Biometrics 1986;42:121-130.

14. Andersen P, Gill R. Cox's regression model for counting processes: a large sample study. Ann Statistics 1982;10: $1100-1120$.

15. Klausner JD, McFarland W, Bolan G, Hernandez MT, Molitor F, Lemp GF, Cahoon-Young, Morrow S, Ruis J, Young Women's Survey Team. Knock-knock: a populationbased survey of risk behavior, health care access, and Chlamydia trachomatis infection among low-income women in the San Francisco Bay Area. J Infect Dis 2001;183:1087-1092.

16. Curtis K, Hillis SD, Kieke B, Brett K, Marchbanks PA, Peterson HB. Visits to emergency departments for gynecologic disorders in the United States, 1992-1994. Obstet Gynecol 1998;91:1007-1012.

17. Centers for Disease Control and Prevention. Sexually transmitted disease surveillance, 2001. Atlanta, GA: US Department of Health and Human Services; 2002.

18. Blythe MJ, Katz BP, Batteiger BE, Ganser JA, Jones RB. Recurrent genitourinary chlamydial infections in sexually active female adolescents.J Pediatr 1992; 21:487-493.

19. Miller JM. Recurrent chlamydial colonization during pregnancy. Am J Perinatol 1998;15:307-309.

20. Allaire AD, Huddleston JF, Graves WL, Nathan L. Initial and repeat screening for Chlamydia trachomatis during pregnancy. Infect Dis Obstet Gynecol 1998;6:116-122.

21. Whittington WLH, Kent C, Kissinger P, Oh MK, Fortenberry JD, Hillis SE, Litchfield B, Bolan GA, St Louis ME, Farley TA, et al. Determinants of persistent and recurrent Chlamydia trachomatis infection in young women: results of a multicenter cohort study. Sex Transm Dis 2001; 28:117-123.

22. Fortenberry JD, Brizendine EJ, Katz BP, Wools KK, Blythe MJ, Orr DP. Subsequent sexually transmitted infections among adolescent women with genital infection due to Chlamydia trachomatis, Neisseria gonorrhoeae, or Trichomonas vaginalis. Sex Transm Dis 1999;26:26-32.

23. Hillis SD, Nakashima A, Marchbanks PA, Addiss DG, Davis JP. Risk factors for recurrent Chlamydia trachomatis infections in women. Am J Obstet Gynecol 1994;170:801-806.

24. Peipert J, Ness R, Blume J, Soper DE, Holley R, Randall H, Sweet RL, Sondheimer SJ, Hendrix SL, Amortegui A, et al. Clinical predictors of endometritis in women with symptoms and signs of pelvic inflammatory disease. Am J Obstet Gynecol 2001;184:856-863. 




The Scientific World Journal
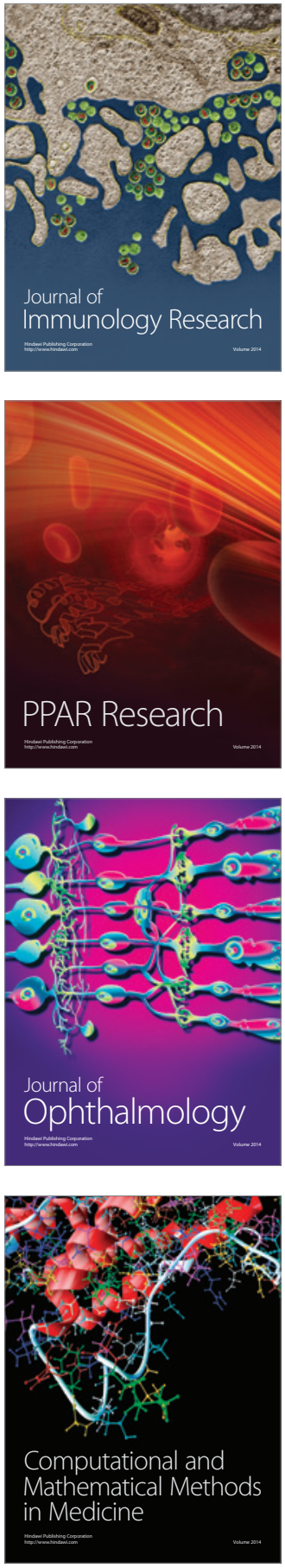

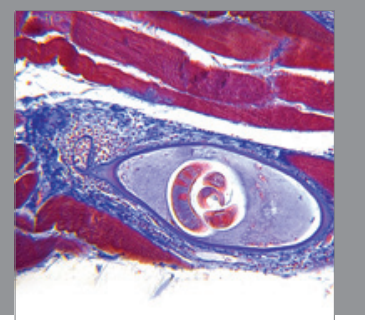

Gastroenterology

Research and Practice
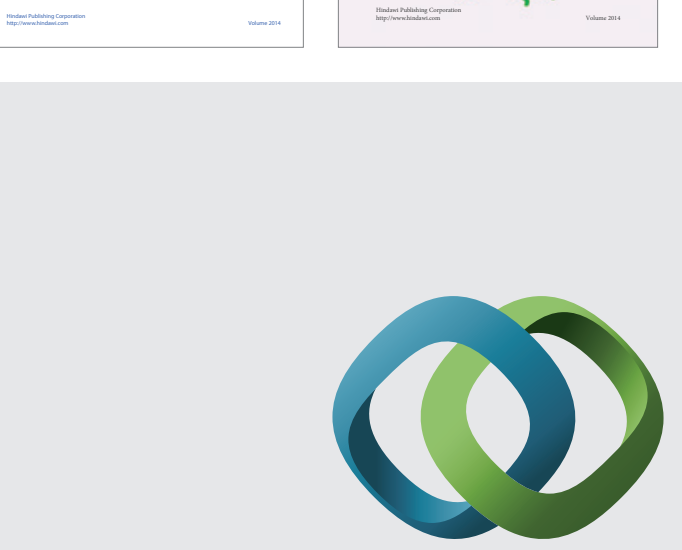

\section{Hindawi}

Submit your manuscripts at

http://www.hindawi.com


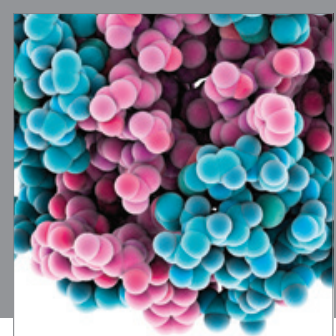

Journal of
Diabetes Research

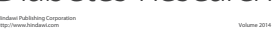

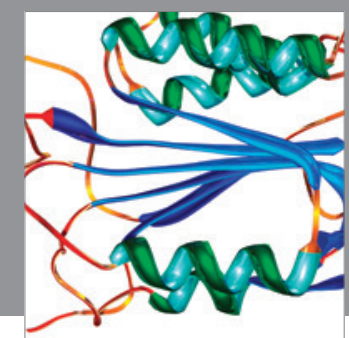

Disease Markers
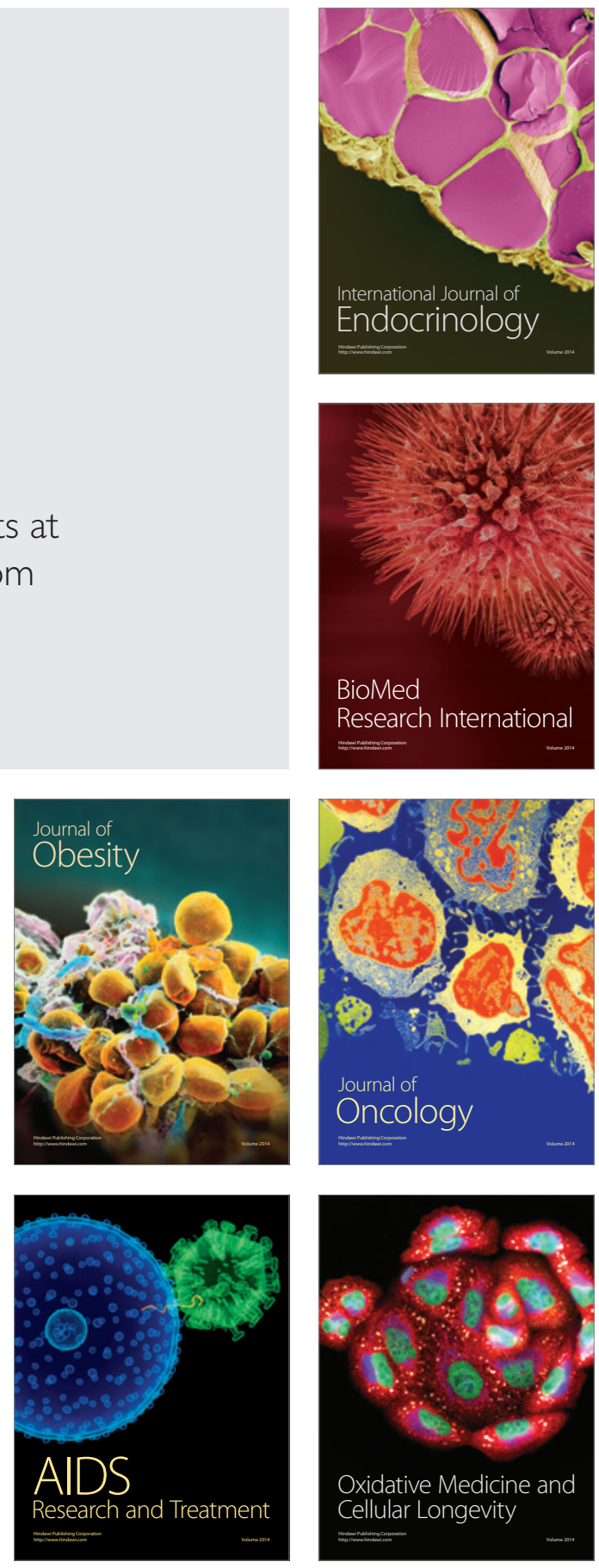